\title{
ORIGINAL
}

\section{D PRINTING IN PEDIATRIC ORTHOPEDICS - THE NEW GENERATION OF PREOPERATIVE PLANNING IN THE FIELD OF PEDIATRIC ORTHOPEDICS}

\author{
Eduard Liciu ${ }^{1,3,5,6}$, Maria Miruna Mihai ${ }^{2,3,5,6}$, Ștefana Carp ${ }^{1,7}$, Laura Popa ${ }^{1}$, Camelia Vreme ${ }^{8}$, \\ Vlad Costel ${ }^{1,4}$ \\ 1 "Victor Gomoiu" Hospital for Children Doctor Bucharest, Romania \\ ${ }^{2}$ Emergency Hospital for Children "Grigore Alexandrescu", Bucharest, Romania \\ 3 "Carol Davila University of Medicine and Pharmacy", Bucharest, Romania \\ 4 "Titu Maiorescu" University of Medicine and Pharmacy, Bucharest, Romania \\ ${ }^{5}$ Centre of Innovation and eHealth UMFCD, Bucharest, Romania \\ 6 "The Romanian Medical Association of 3D Biomodelling" - AMRB 3D Bucharest, \\ Romania \\ 7 "Grigore T Popa" University of Medicine and Pharmacy, Iași, Romania \\ 8 "Maria Sklodowska Curie" Emergency Hospital for Children, Bucharest, Romania \\ Corresponding author: Eduard Liciu \\ E-mail: eduard.liciu@drd.umfcd.ro
}

\begin{abstract}
The evolution of modern medicine, in its continuous developing process, is highly connected with the progress achieved in the medical branch of technology. Regarding the surgical specialties, the technological progress breakthroughs may determine the appearance of new diagnosis techniques, but also shape innovative treatments, leading to superior therapeutic results. In the surgical treatment as a whole, an essential role is played by the Medical Imagistics. They either offer the much-needed visual support in order to reach an accurate diagnosis, or guide the surgeon in choosing a certain type of intervention. The importance of Imagistics is indisputable. It has also been proven so in intraoperatory guidance and monitoring the patient in post-surgery. In the evolution of medical Imagistics, after the transition to digital imaging, followed by graphic $3 D$ reconstructions based on $C T$ and MRI data, we find ourselves contemporary with a new turning point announcing a technological revolution: the transition from virtual $3 D$ models to tangible $3 D$ replica. Since the beginning, the $3 D$ printing technology has been of great importance to the field of medical research and, once the technique gained popularity, it became a modern tool for many medical specialties, in particular for cranio-maxillofacial surgery, orthopedics, oncology, neurosurgery. The 3D printing technology managed to transgress dated barriers by facilitating the manufacturing of implants or implement new treatments in regenerative medicine. The purpose of this original paper is to present our $3 D$ printing work protocol and general conclusions after 5 years of implementing $3 D$ printing in pediatric orthopedics.
\end{abstract}

Keywords: 3D printing, orthopedic pediatric pre-surgical planning, personalized treatment 


\section{Introduction}

Medical Imagistics provides tools of significant importance that facilitate the work of a pediatric orthopedic surgeon, such as visual support essential in establishing the diagnosis, but also equipment for examination that allows pre-surgical planning (CT-3D, IRM- 3D), intraoperatory guidance (C-Arm, O-Arm, Spine Navigation), monitoring patients postintervention, or enabling a long-term surveillance. The ascending development of this medical branch leads to groundbreaking acquisitions in the medical field, such as digitalization, 3D virtual reconstructions and so on. However, there are few disadvantages:

- The Spine Navigation System requires great financial costs of use; it implies a high dose of radiations issued during surgery and it also increases the intraoperative time [1];

- 3D reconstructions viewed on 2D devices (monitors, TVs, tablets) limit the information regarding the segment being analyzed. The consequence lies in the fact that the doctor must assemble a cognitive and subjective $3 \mathrm{D}$ reconstruction based on a sequence of 2D images. This way, among the members of the operative team, there may occur a difference of opinion regarding the subjective nature of an individual virtual 3D reconstruction. (Figure 1).

Lately, 3D printing proved to be a solution to all these problems, by offering the possibility of converting virtual $3 \mathrm{D}$ reconstructions into tangible 3D printed models [2-5]. 3D printers have now become widely available and inexpensive, and they can be used to rapidly produce life-size models based on CT scans of a patient [6]. Using these 3D printed models, it is now possible to configure pre- operative planning, with multiple benefits, such as increasing the intervention's success rate, decreasing the number of reinterventions required [7] and reducing intra-operative time and blood loss [8]. The purpose of this original paper is to present our 3D printing work protocol and general conclusions after 5 years of implementing 3D printing in pediatric orthopedics.

\section{Materials and Methods}

A multidisciplinary team (made out of an orthopedic surgeon, a radiologist and an IT technician) is essential to allow the use of 3D printing Technology in a presurgical planning purposes $[6,9,10]$. Our team consisted only in a radiologist and an orthopedic surgeon but both have skills in using visual graphic design software. This proved to be a real advantage because it leads to a shorter time spent on data processing.

Our process involving the use of 3D printing for pre-operative planning purposes describes the following steps:

- The acquisition of diagnostic imaging data. The CT data need to have certain parameters for 3D printing purposes. Slice thickness needs to be equal or less than $1 \mathrm{~mm}$ and the Kerner parameter needs to be set to B40s (medium). The data collected using these parameters will then be exported in a DICOM format (Digital Imaging and Communications in Medicine)

- The DICOM files will then be processed using different software: 3D Slicer [11] and Osirix [12]. The processing steps are (Figure 2)

- 3D data presentation - 3D Slicer volume rendering- Using DICOM images the software is able to make a $3 \mathrm{D}$ reconstruction

- Interest cropping region - Volume extraction for the purpose of having a region of interest on the bone geometry

- Segmentation - Applying a threshold using the Hounsfield scale to select only the bone structures 


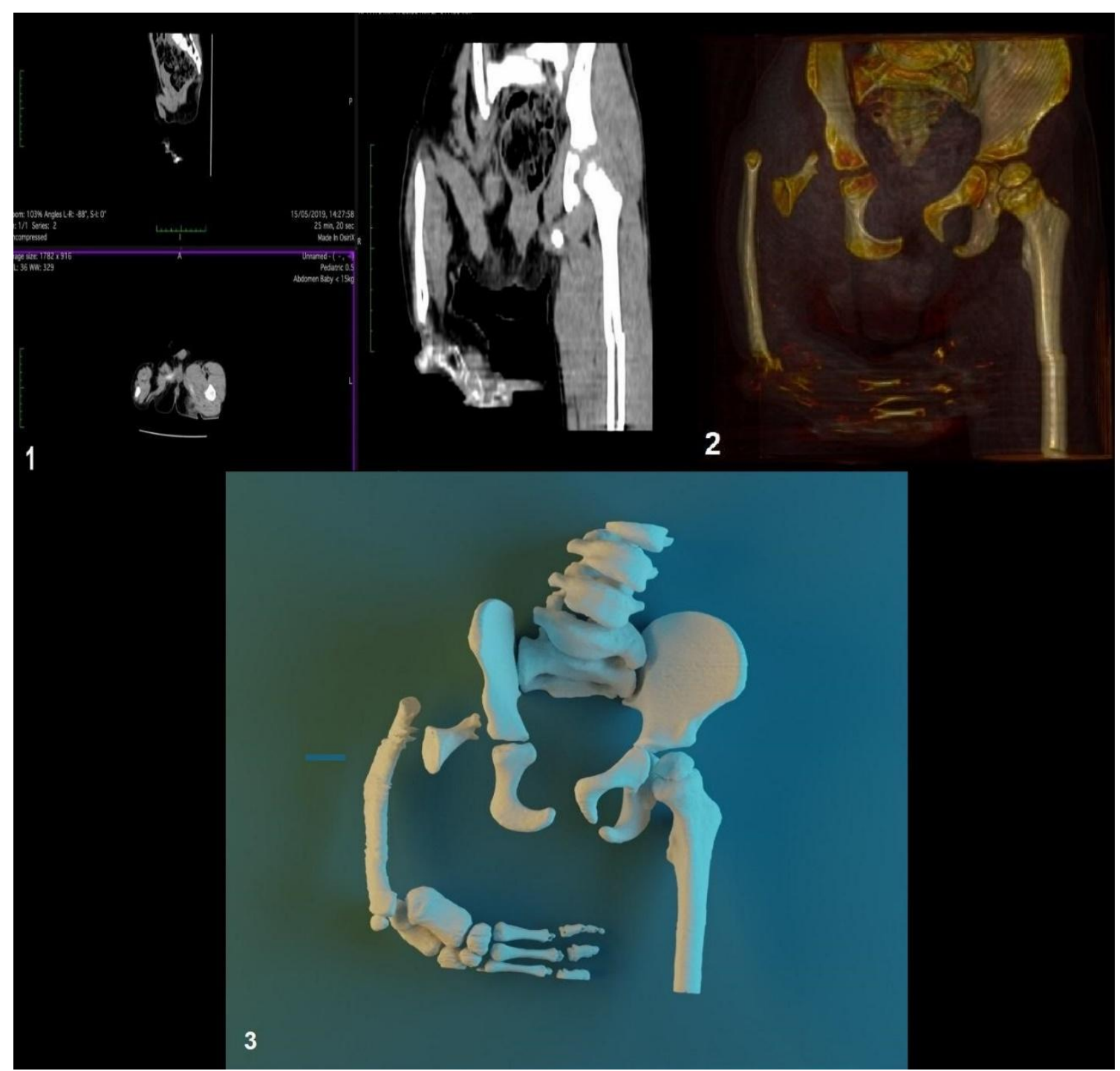

Figure 1 - Patient selected from our cases with sever congenital malformations of the hip and limb Agenesis of the right pubis, femoral hypoplasia, agenesis of the right tibia. CT-scan images analysis (1), 3D Volume rendering reconstruction (2), virtual 3D reconstructions (3).

Exporting the file (bone structure) in a Standard Triangle Language format (.stl format). This file format can be read by a $3 \mathrm{D}$ printing software [13]

- This .stl file will now be processed more in an open-source software called Blender 2.79 [14]. Here, errors like isolated parts of a mesh, non-manifold triangles, edges and vertices will be removed [13] (Figure 3). If the model is too large and the printer cannot print the whole model in one piece, Blender allows us to section our model into smaller parts that can be printed separately and then assembled post-printing. This provides the following advantages: lower printing times and less material used.
- This .stl file/files will now be exported in Autodesk MeshMixer [15] for some "cleaning" processes like closing holes in the model and removing isolated vertices.

- Creating a G-code. We export the stl file to Cura software [16] where we choose our printing parameters depending on what material we want to use (in our case, a filament of PLA with $1,75 \mathrm{~mm}$ diameter): Layer Height $0.1 \mathrm{~mm}$, Wall Thickness $0.80 \mathrm{~mm}$, Fill Density 18\%, Speed Print $50 \mathrm{~mm} / \mathrm{s}$, Speed Travel 150 $\mathrm{mm} / \mathrm{s}$, Speed Infill $85 \mathrm{~mm} / \mathrm{s}$, etc.

- Scaling and positioning our 3D model on the printing bed.

- 3D printing (using a Creality Ender 3 Pro 3D Printer). 


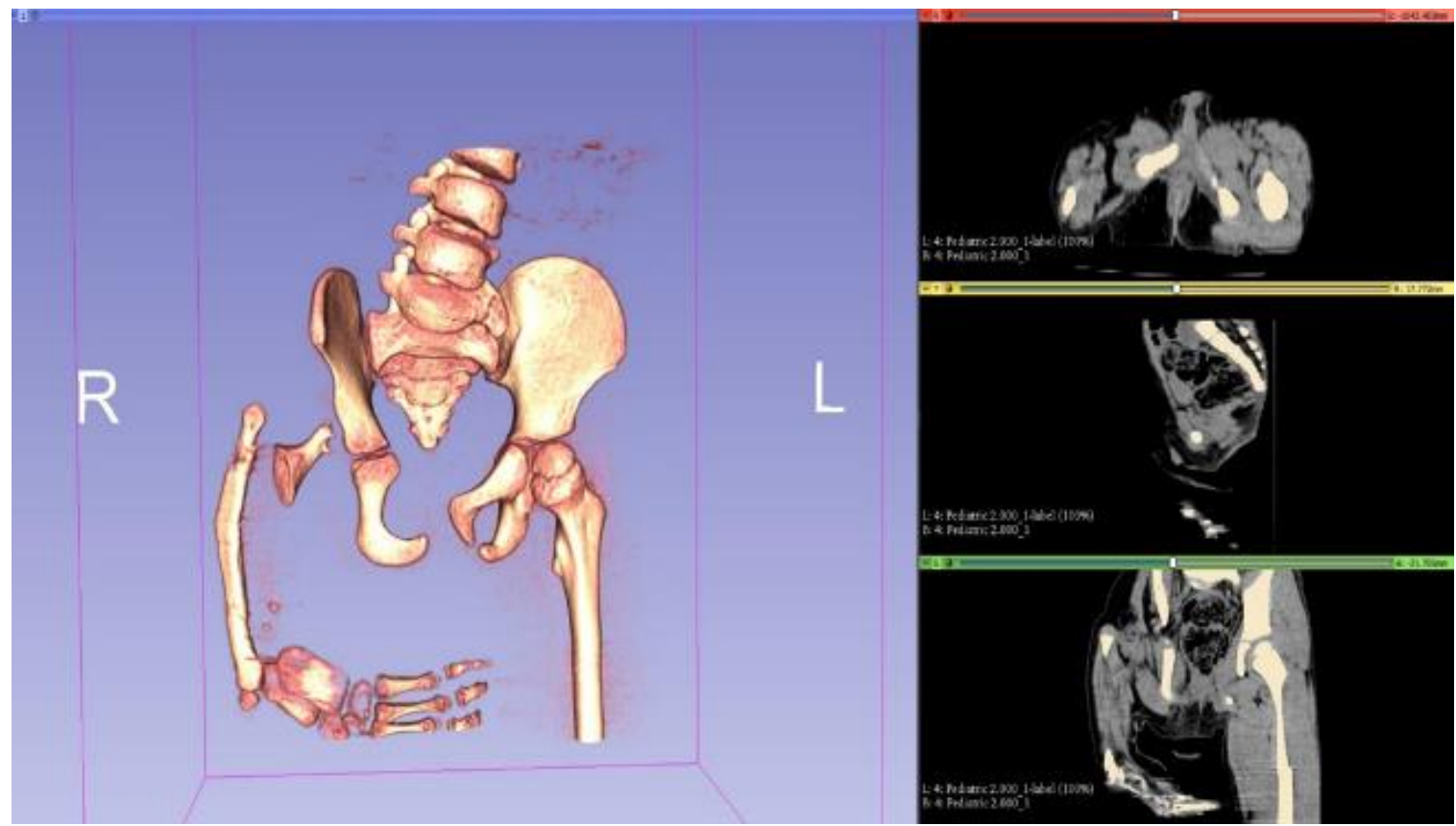

Figure 2 - The underlining and the "extraction" of the bone geometry from targeted DICOM files with 3D Slicer

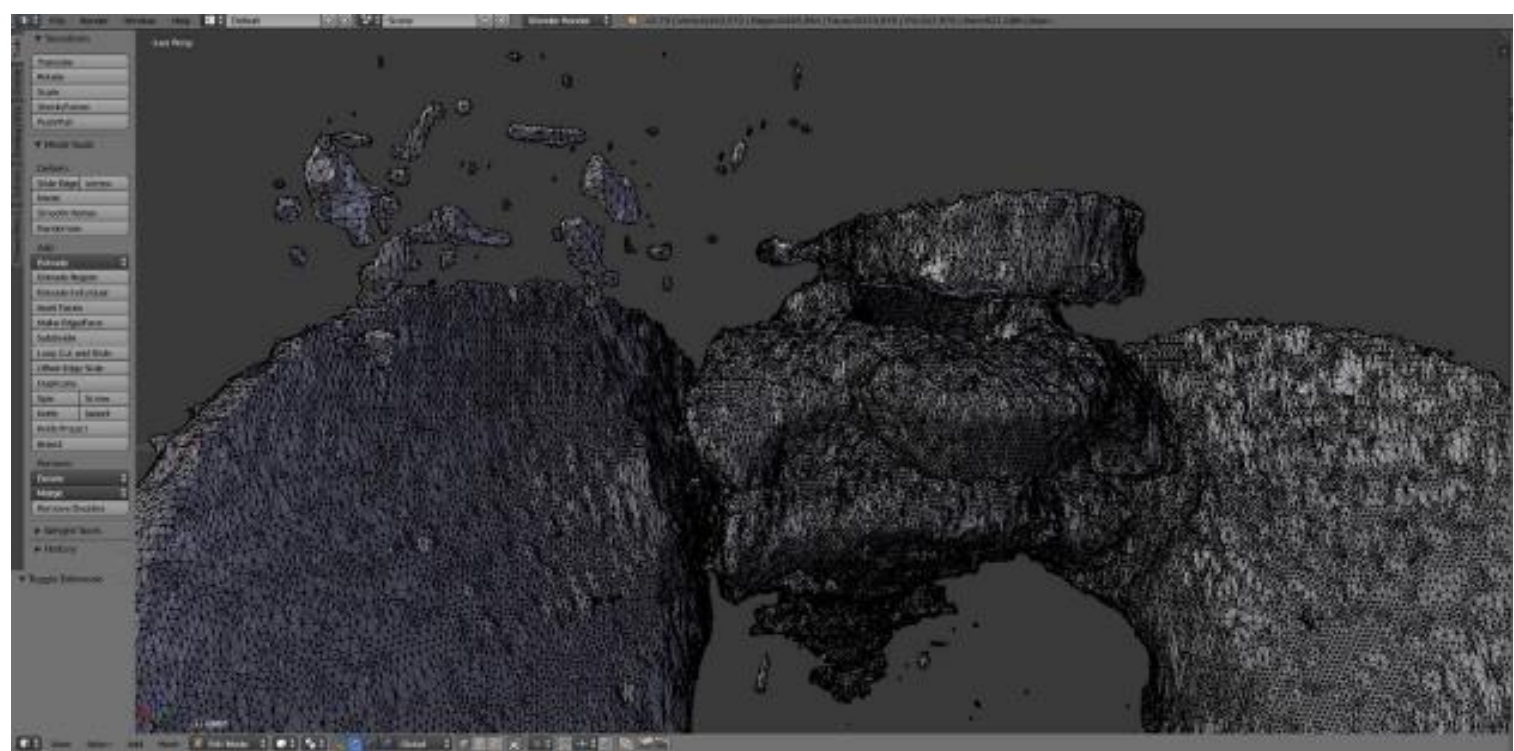

Figure 3 - 3D graphic model of a pelvis with hip dysplasia - before the cleaning process in Blender 3D

\section{Results}

\section{Our 3D printing medical experiences}

The 3D printing technology could be applied in the treatment of a vast number of medical cases. Printing in 3D based on the DICOM information influences many aspects of pediatric orthopedics, such as the approach of preoperative planning, choosing the surgical technique and it can also influence the intraoperative settings. These uses could be transposed into understanding the anatomical particularities of the concerned region by examining the segment's deformities and bone malformations (e.g., spine deformities [17-20], long bone malformations, post traumatic bone squeal, arthritis sequelae). Among others, its uses include making accurate measurements on the 3D palpable model (such as pedicle evaluation for screw insertion in surgical treatment of scoliosis, acetabulum quantification in preoperative planning for the hip arthroplasty [21] etc.) and establishing and simulating the osteotomy trails in various pathologies and choosing and customizing of the osteosynthesis material (vertebrectomy planning in spine malformation [20] etc.). In the cases of malignant 
bone tumors, a sterilizable 3D printed complementary tool which is the same size as the bone tumor (as measured on CT) plus the oncological safety resection margins could be created and used as an intraoperative guide mark in helping to discuss the oncological safety limits during tumor resection [22].

In spine deformities, such as scoliosis regardless of the initial trigger that leads to the installation of spinal curvature, axial loading produces an asymmetric unloading of

the biomechanical forces upon the vertebrae that are responsible for most, if not of all the progression of spinal curvature [23-37] and secondary vertebral deformation, which can be better studied and understood by using 3D printed replicas (Figure 4).

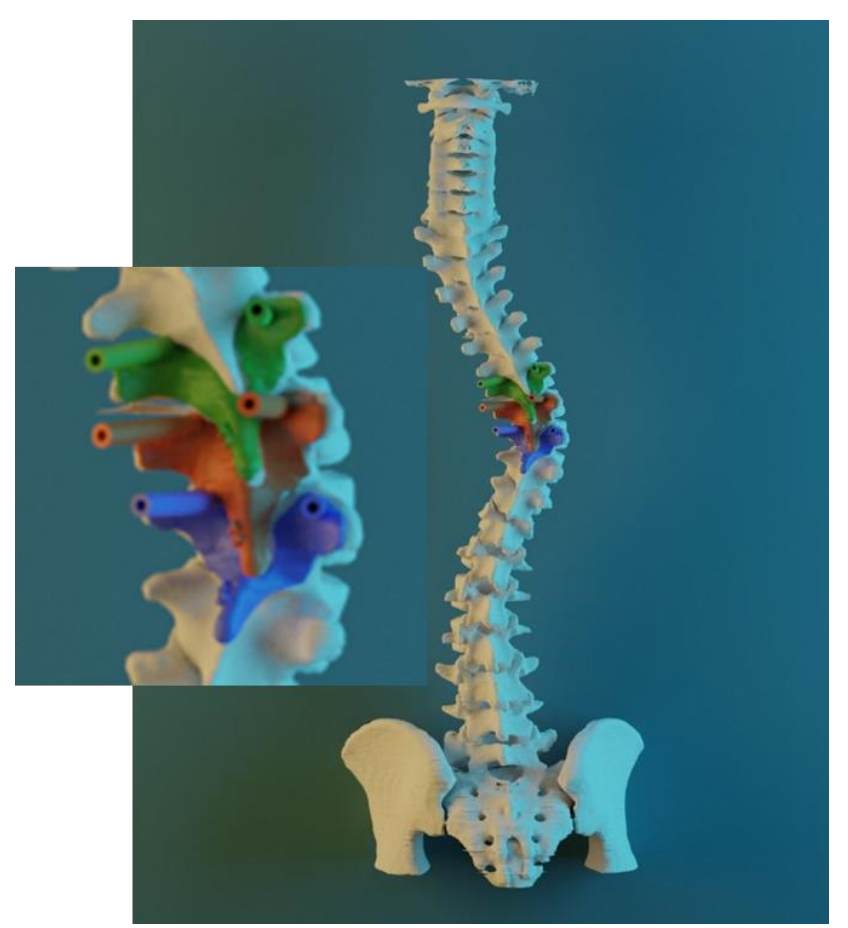

Figure 4-3D printed spine in a case of scoliosis. Attached to the spine there are the custom devices designed and made by our team members that serve for surgical guidance. The model has been used with great success in the preoperative planning process and also for intraoperative guidance

In this kind of pathology, the technique could be implemented for the preoperative planning (e.g., in kypho-scoliosis it can be used for vertebral counting, identification of vertebral malformations such as hemivertebrae or vertebral blocks, choosing the vertebrectomy levels [38], planning the instrumentation levels, the pedicle entry-points and trajectories of pedicle screws and the identification of the posterior vertebral elements in order to be resected during spinal arthrodesis.

\section{Educational purpose}

Practicing the surgical maneuvers constitutes an essential part of the learning process for the young surgeon during his or her training that comes as a complementary factor alongside the theoretical aspect of the knowledge required. Lately, the ethical aspect of the fact that the surgeon must perform his or her first surgical attempts on a live patient has been disputed more and more often. Regarding this issue, the 3D Printing Technology offers a new angle to the problem by developing a solution to it. This way, a 3D tangible and personalized replica of the patient's body segment undergoing surgery can be provided to the apprentice surgeons to practice their abilities before entering surgery. This privilege does not only concern the improvement of their current knowledge or the expand of it, but it also offers a psychological comfort that empowers the young surgeon to a better assimilation of the surgical maneuver which is about to be acquired.

The 3D printed models are a faithful reproduction of the patient's target body segment, incorporating it's particular anatomical and pathological features giving the possibility of serving in the academic field. The 3D Printing Technology managed to bridge the gap between digital and physical, opening a new path in the medical educational system. The advantages of bringing objects from a computer, a textbook or an atlas and more importantly from a patient's body in the hands of students for inspection, observation, analysis or any other process that can benefit from physical manipulation, are multiple. Apart from the resilience and the ability to preserve the original's features, the printed model offers the chance to explore the segment's geometry in 3D full body $360^{\circ}$, making its self-easy to manipulate due to its lightweight and portable characteristic.

\section{Malpractice prevention}

The printed models obtained through this new technology combined with medical Imagistics constitute an exceptional tool enabling a simulation of the intervention's surgical steps and maneuvers performed on a model that 
preserves the anatomical particularities of the patient undergoing surgery. This way, the risk of surgical errors is significantly reduced due to the pre-intervention practice conducted in safe conditions.

A different purpose that this 3D printed model can serve is facilitating the doctor-patient relationship, proving to be a mediator between the doctor's knowledge and explanations and the patient's level of comprehension, limited by different educational background. By not fully understanding the pathology in question, or the changes that their bodies undergo, patients have the tendency to have high, unrealistic hopes regarding the prognosis, the recovery and the surgical risks. It can also be sometimes hard for them to comprehend that their pathologyinduced changes limit the therapeutical act.

Therefore, the use of 3D replicas becomes a powerful tool in explaining the informed consent. Materializing an abstract concept, it leads to a better understanding of the patient's ailment or treatment along with a reduced level of insecurities on the patient's behalf towards the medical act [39].

\section{Discussions}

Patient uniqueness, surgeon training and decision making

In surgery, like in other human activities, every action is preceded by a decision making. The quality of our actions depends on the speed and precision of decision making. Understanding the mechanisms of decision making may enhance the results of the surgery. Planning the operation is one key-step, especially in orthopedic surgery. There are at least two dimensions: the uncertainty and the risk. The uncertainty results from that in real world things never happens one hundred percent as planned, in other words anticipating all possible scenarios need to analyze a huge amount of data. Risk emerges from the bad result probability or lifethreatening situation during surgery. The response to the two challenges relies on the speed and quality of decision making. Let's think to the connection between hand movements and brain commands of the surgeon and compare with what happens when a football player catches a ball. It is not about conscient trajectory calculation taking in account wind speed, air temperature, ground altitude and many other variables; it is about training and intuition. The intuition may be trained. The orthopedic surgery is one field in which 3D replica of the human body parts will definitely train the surgeon's mind to take better decision during the unique moments of surgery. Studying the 3D replica will train the eye, the amplitude of the hand movement, and will improve the implant selection. Learning the unique bony landmarks of the patient will allow the surgeon to be less aggressive with the soft tissues. Implant placement will be more accurate, the confidence of the surgeon will improve, the negative emotions during surgery will diminish and this chain reaction will generate a better outcome for the patient.

\section{Conclusion}

Introducing the 3D Printing Technology in the pre-operative planning steps, involved in the treatment of various orthopedic pathologies, leads to a better understanding of the disease and deformity, therefore improving the treatment plan.

The opportunity of studying the anatomical particularities, in order to establish the optimal surgical approach and also choose the proper surgical instruments and implants for osteosynthesis, comes with a real benefit for the patient as well. Not only a lower rate of intraoperative risk is achieved by using this technology, but also it leads to a more competent way of explaining the pathology and the informed consent to the patient.

According to medical literature, numerous clinical cases using this technology, especially in complex pathologies were reported as successful. For educational purposes, in training junior doctors, 3D printing plays an important role, allowing the simulation of surgical gestures on a custom model which represents the exact replica of the patient's bone anatomy.

Acknowledgements: This article was possible due to a close collaboration with the Center of Innovation and e-Health UMFCD, Bucharest, Romania. 


\section{References}

[1] Y Ito, Y Sugimoto, M Tomioka, Y Hasegawa, K Nakago and Y Yagata: Clinical accuracy of 3D fluoroscopy-assisted cervical pedicle screw insertion. J Neurosurg Spine (2008) 9; 450-453.

[2]GA Brown, K Firoozbakhsh, TA DeCoster, JR Reyna Jr, M Moneim: Rapid prototyping: the future of trauma surgery? J Bone Surg Am 2003. 85-A Suppl 4: 49-55.

[3] PS D’Urso, G Askin, JS Earwaker, Merry GS, Thompson RG,Baker TM, Effeney DJ: Spinal biomodeling. Spine 1999, 24: 1247-1251.

[4] M Yamazaki, A Okawa, R Kadota, C Mannoji, T Miyashita, $M$ Koda: Surgical simulation of circumferential osteotomy and correction of cervicothoracic Kyphoscoliosis for an irreducible old C6-C7 fracture dislocation. Acta Neurochir (Wein) (2009) 151: 867-872.

[5] J Mizutani, T Matsubara, M Fukuoka, N Tanaka, H Iguchi, A Furuya, H Okamoto, I Wada, T Otsuka: Application of full-scale three-dimensional models in patients with rheumatoid cervical spine. Eur Spine J (2008) 17: 644-649

[6] JM Duncan, S Nahas, K Akhtar, J Daurka: The Use of a 3D Printer in Pre-operative Planning for a Patient Requiring Acetabular Reconstructive Surgery. Journal of Orthopaedic Case Reports 2015 Jan-March: 5(1): Page 23-25.

[7] F Auricchio, S Marconi: 3D printing: Clinical applications in orthopaedics and traumatology, EFORT open reviews, EOR, volume 1, May 2016, 121-127.

[8] YU AW, JM Duncan, JS Daurka, A Lewis, J Cobb, A Feasibility Study into the Use of ThreeDimensional Printer Modelling in Acetabular Fracture Surgery; Hindawi Publishing Corporation Advances in Orthopedics, Volume 2015, Article ID 617046, 4 http://dx.doi.org/10.1155/2015/617046.

[9] I Ono, K Abe, S Shiotani, Y Hiramaya. Producing a full-scale model from computed tomographic data with the rapid prototyping using the binder jet method: a comparison with the laser lithohraphy method using a dry skull. J Craniofac Surg 2000;11(6):527-537.

[10] E Huotilainen, M Paloheimo, M Salmi, et al. Imaging requirements for medical applications of additive manufacturing. Acta Radiol 2014;55(1):7885.

[11] A Fedorov, R Beichel, J Kalpathy-Cramer, J Finet, JC Fillion-Robin, S Pujol, C Bauer, D Jennings, FM Fennessy, M Sonka, J Buatti, SR Aylward, JV Miller, S Pieper, R Kikinis: 3D Slicer as an Image Computing Platform for the Quantitative Imaging
Network. Magn Reson Imaging. 2012 Nov;30(9):1323-41.

[12] OsiriX: www.osirix-viewer.com

[13] S Widel, A Szczesna, A Widel, D Spinczyk: Orthopedic pre-surgical planning using a 3D printed model, Studia Informatica, Volume 37, Number 3B (126), 2016.

[14] GNU General Public License, Blender 2.79, release September 11, 2017, https://www.blender.org/.

[15] Autodesk® Meshmixer, Autodesk MeshMixer is a registered trademark of Autodesk, Inc., and/or its subsidiaries and/or affiliates in the USA and other countries, https://www.meshmixer.com/.

[16] Copyright (C) 2012 WANHAO 3D PRINTER. All Rights Re, WanhaoMaker 2.3.5.2241, http://www.wanhao3dprinter.com/.

[17] Y Ito, Y Sugimoto, M Tomioka, Y Hasegawa, K Nakago, Y Yagata: Clinical accuracy of 3D fluoroscopy-assisted cervical pedicle screw insertion. J Neurosurg Spine (2008) 9; 450-453;

[18] GA Brown, K Firoozbakhsh, TA DeCoster, JR Reyna Jr, M Moneim: Rapid prototyping: the future of trauma surgery? J Bone Surg Am 2003. 85-A Suppl 4: 49-55.

[19] PS D'Urso, G Askin, JS Earwaker, GS Merry, RG Thompson, TM Baker, DJ Effeney: Spinal biomodeling. Spine 1999, 24: 1247-1251;

[20] M Yamazaki, A Okawa, R Kadota, C Mannoji, $\mathrm{T}$ Miyashita, $\mathrm{M}$ Koda: Surgical simulation of circumferential osteotomy and correction of cervicothoracic Kyphoscoliosis for an irreducible old C6-C7 fracture dislocation. Acta Neurochir (Wein) (2009) 151: 867-872;

[21] JS Matsumoto, JM Morris, TA Foley et al, Three-dimensional Physical Modeling: Applications and Experience at Mayo Clinic; RadioGraphics, vol 35(7):1989-2006;

[22] M Limin, Z Ye, Z Ye, L Zefeng, W Yingjun, Z Yu, Hong Xia \&Chuanbin Mao: 3D-printed guiding templates for improved osteosarcoma resection, Published: 21 March 2016, www.nature.com/scientificreports;

[23] J Antaniou, V Arlet, T Goswami, M Aebi, M Alini: Elevated synthethic activity in the convex side of scoliotic intervertebral disc and endplates compared with normal tissues. Spine 2001, 26: E 198 - E 206.

[24] B Cheng, J Fellenberg, H Wang, C Carstens, W Richter: Occurence and regional distributin of apoptosis in scoliotic discs. Spine 2005. 30: 519-524. [25] T Kluba, T Niemeyer, C Gaissmaier, T Grunder: Human anulus fibrosis and nucleus pulposus cells of the intervertebral disc - Effect of degeneration and culture system on cell phenotype. Spine 2005, 30: 2743-2748. 
[26] KG Shea, T Ford, RD Bloebaum, J D'Astous, H King: A comparison of the microarchitectural bone adaptations of the concave and convex thoracic spineal facets in IS. J Bone Jt Surg 2004, 86-A 10001006.

[27] MR Urban, JCT Fairbank, SRS Bibby, JPG Urban: Intervertebral disc composition in neuromuscular scoliosis. Change in cell density and glycosaminoglycan concentration at the curve apex. Spine 2001, 26: 610-617.

[28] I Villemure, CE Aubin, G Grimard, J Dansereau, $\mathrm{H}$ Labelle: Progression of vertebral and spinal 3D deformities in AIS. A longitundinal study. Spine 2001, 26: 2244-2250.

[29] I Villemure, MA Chung, CS Seck, MH Kimm, JR Matyas, NA Duncan: The effects of mechanical loading on the mRNA expression of grwth-plate cells. Research into Spinal Deformities 2002, 4: 114-118. [30] R Roaf: Vertebral growth and its mechanical control. J Bone Jt Surg 1960, 42B: 40.

[31] IAF Stokes: Hueter-Volkmann Effect. Spine: State of the Art Reviews 2000, I4: 349-357.

[32] MG Gardner-Morse, IAF Stokes: Trunk stiffness increases with steady state effort. J Biomechanics 2001, 34: 457-463.

[33] PL Mente, DD Aronsson, IAF Stokes, JC Iatridis: Mechanical modulation of growth for the correction of vertebral wedge deformities. J Orthop Res 1999, 17: 518-524.

[34] IAF Stokes, DD Aronsson: Disc and vertebral wedging in patients with progressive scoliosis. J Spinal Disorders 2001, I 4: 317-322.

[35] IAF Stokes, MG Gardner-Morse: Muscle activation strategies and spinal loading in the lumbar spine with scoliosis. Spine 2004, 2009: 2103-2107.

[36] IAF Stokes, H Spence, DD Aronsson, N Kilmer: Mechanical modulation of vertebral body growth. Implications for scoliosis progression. Spine 1996, 21: 1162-1167.

[37] MH Pope, IAF Stokes, M Moreland: The biomechanics of scoliosis. Crit Rev Biomed ENG 1984, I 1:157-188.

[38] Y Sugimoto, M Tanaka, R Nakahara, H Misawa, $\mathrm{T}$ Kunisada, $\mathrm{T}$ Ozaki: Surgical treatment for congenital Kyphosis corection using both spinal navigation and 3D model, Acta Med. Okayama 2012, Vol. 66, No.66: 499-502.

[39] I Tevanov, E Liciu, MO Chirila, A Dusca, Al Ulici. The use of 3D printing in improving patientdoctor relationship and malpractice prevention, Rom J Leg Med [25] 279-282 [2017] DOI: 10.4323/rjlm.2017.279. 